\title{
RADIAL LIMITS OF QUASICONFORMAL FUNCTIONS
}

\author{
D. A. STORVICK ${ }^{11}$
}

Beurling and Ahlfors [1] answered a fundamental question concerning the boundary correspondence induced by a quasiconformal mapping when they proved that the correspondence need not be given by an absolutely continuous function. They proved this by characterizing the boundary correspondences of quasiconformal mappings of the upper half-plane $\operatorname{Im}(z)>0$ onto the upper half-plane $\operatorname{Im}(w)>0$ under which the boundary points at infinity correspond. They proved that a necessary and sufficient condition that the strictly monotone increasing function $\mu(x)$ carrying the real axis onto itself be the boundary correspondence induced by such a quasiconformal mapping is that $\mu(x)$ should satisfy a $\rho$-condition

$$
\frac{1}{\rho} \leqq\left[\frac{\mu(x+t)-\mu(x)}{\mu(x)-\mu(x-t)}\right] \leqq \rho
$$

for some constant $\rho$ and all real $x$ and $t$. In addition they showed that if the $\rho$-condition is fulfilled there exists a quasiconformal mapping whose dilatation does not exceed $\rho^{2}$ and every mapping with the boundary correspondence $\mu(x)$ must have a maximal dilatation greater than or equal to $1+A \log \rho$ where $A$ is a certain numerical constant $(=.2284)$. For a given mapping $\mu(x)$ of the real axis denote by $\rho(\mu)$ the smallest value of $\rho$ such that the $\rho$-condition (1) is fulfilled. Beurling and Ahlfors showed the stronger result that there exists a quasiconformal mapping of the half-plane onto itself whose boundary correspondence is given by a completely singular function $\mu(x)$ with $\rho(\mu)$ arbitrarily close to one. Because of this result and its function-theoretic consequence that the distinction between sets of zero and positive harmonic measure is not preserved under quasiconformal mappings, it follows that the analogue of Fatou's theorem does not hold for quasiconformal functions. The purpose of this note

Received June 20, 1963.

1) Sponsored by Contract No. AF 49 (638)-836 with the AFOSR and by the Mathematics Research Center, U.S. Army, University of Wisconsin under Contract DA-11-022ORD-2059. 
is to show that the boundary correspondence induced by a quasiconformal mapping is absolutely continuous and an analogue of Fatou's theorem is valid if one requires that the dilatation quotient in addition to being bounded, tends to one sufficiently swiftly as the boundary is approached.

Let $w=f(z)$ denote a sense-preserving homeomorphism of a domain $\Omega$ in the $z=x+i y$ plane onto a domain $\Omega^{\prime}$ in the $w=u+i v$ plane. Let $D$ be any Jordan domain contained together with its boundary curve within $\Omega$, and let $z_{1}$, $z_{2}, z_{3}, z_{4}$ be any four distinct boundary points of $D$ which lie in this order on the positively oriented boundary curve ${ }^{1)}$. By means of a conformal mapping, map $D$ onto a rectangle:

$$
0<\xi<1,0<\eta<h \text { in the } \zeta=\xi+i \eta
$$

plane sending the points $z_{1}, z_{2}, z_{3}, z_{4}$ onto $0,1,1+i h$, ih respectively. The number $h$ which is uniquely determined by $D$ and the $\left\{z_{i}\right\}$ is defined as the modulus of $D$. We shall assume that there exists a constant $K \geqq 1$ such that for all Jordan domains $D \subset \Omega$, the modulus of $f(D)$ is less than or equal to the modulus of $D$ :

$$
\bmod f(D) \leqq K \bmod (D) \text {. }
$$

Such a transformation $w=f(z)$ is a $K$-quasiconformal mapping of the domain.

It has been proved by Mori [7] that a $K$-quasiconformal mapping is totally differentiable at almost all points in $\Omega$ and at every point at which it has a total differential,

$$
\max _{\theta}\left|D_{\theta} w\right|^{2} \leqq K \cdot J(z)
$$

where $D_{\theta} w=\left(u_{x} \cos \theta+u_{y} \sin \theta\right)+i\left(v_{x} \cos \theta+v_{y} \sin \theta\right)$ and $J(z)=u_{x} v_{y}-v_{x} u_{y} \geqq 0$ and for almost all $y=y_{0}, w=f\left(x+i y_{0}\right)$ is absolutely continuous in $x$ on any closed interval contained in the intersection of $y=y_{0}$ and $\Omega$.

At points where the mapping is totally differentiable there is defined the dilatation quotient, $Q[f(z)]$, which describes the local distortion of the mapping;

$$
Q[f(z)]=\frac{\max _{\theta} D_{\theta}(f(z))}{\min _{\theta} D_{\theta}(f(z))},
$$

1) Some authors call such a configuration, $D$ and the points $\left\{z_{i}\right\}$, a quadrilaterai, see [5] and [7]. 
and

$$
Q[f(z)] \leqq K .
$$

We now prove a theorem giving conditions under which the boundary correspondence induced by a quasiconformal mapping is differentiable.

Theorem:

Let $w=f(z)$ be a K-quasiconformal mapping of the upper half-plane $\operatorname{Im}(z)$ $>0$ onto the upper half-plane $\operatorname{Im}(w)>0$. Let there be an essentially bounded measurable function $\kappa(x)$ such that for all $z=x+i y$ for which $Q[f(z)]$ exists,

$$
Q[f(z)]-1 \leqq \kappa(x) \cdot y .
$$

Then at every point $z=x$ on the real axis, the mapping $w=f(z)$ has a complex derivative,

$$
\left.\frac{d f}{d z}\right|_{z=x}=\lim _{\substack{\Delta z \rightarrow 0 \\ \lim \Delta z \geq 0}}\left[\frac{f(x+\Delta z)-f(x)}{\Delta z}\right]
$$

which is different from 0 and $\infty$.

Froof. The mapping $w=f(z)$ can be extended to give a homeomorphism between $\operatorname{Im}(z) \geq 0$ and $\operatorname{Im}(w) \geq 0$ which is real and monotonically increasing on the real axis. Defining $f(z)$ in the lower half-plane by the formula $f(z)=\overline{f(\bar{z})}$, one obtains a quasiconformal mapping of the complex plane onto itself.

Let us now consider $w=f(z)$ in $|z|<1$ and the image $\Delta$ of $|z|<1$ under $f(z)$. We map $\Delta$ in a $1-1$ conformal fashion onto $|\zeta|<1$ by means of $\zeta=$ $g(w)$, normalized so that $g(f(0))=0, g^{\prime}(f(0))>0$. The composition mapping $\zeta=F(z)=g(f(z))$ is a $K$-quasiconformal mapping of $|z|<1$, one-to-one onto $|\zeta|<1$, with $F(0)=0$. Since the dilatation quotient is a conformal invariant, it follows that for the mapping $\zeta=F(z)$, the dilatation quotient satisfies the same inequality as $Q[f(z)]$, i.e.,

$$
Q[F(x+i y)]-1 \leqq \kappa(x) \cdot y
$$

for almost all points in $x^{2}+y^{2}<1$. From the symmetry of the integrand, it follows that for $r<1$,

$$
\int_{0}^{r} \int_{0}^{2 \pi} \frac{Q\left[F\left(\rho e^{i \rho^{\prime}}\right)\right]-1}{\rho} d \rho d \varphi
$$




$$
\begin{aligned}
& \leqq 4 \int_{0}^{r} \int_{0}^{v \sqrt{r^{2}-x^{2}}} \frac{\kappa(x) \cdot y}{x^{2}+y^{2}} d y d x \\
& =2 \int_{0}^{r} \kappa(x)\left(\log r^{2}-\log x^{2}\right) d x \\
& =\int_{0}^{r} H(x) d x .
\end{aligned}
$$

The function $H(x)$ is the product of an essentially bounded measurable function and an integrable function and is therefore integrable. From the absolute continuity of the integral $\int_{0}^{r} H(x) d x$ it follows that there exists a function $\lambda(r)$ where $\lambda(r) \rightarrow 0$ as $r \rightarrow 0$ and

$$
\int_{0}^{r} \int_{0}^{2 \pi} \frac{Q\left[F\left(\rho e^{i \varphi}\right)\right]-1}{\rho} d \rho d \varphi \leqq \lambda(r) .
$$

From a recent result of Lehto [6] it follows that there exists a constant $\gamma^{\prime}, \gamma^{\prime}$ $\neq 0, \infty$, such that $\lim _{z \rightarrow 0} \frac{F(z)}{z}=\gamma^{\prime}$. Since $\zeta=g(w)$ is a conformal mapping, there exists a constant $\gamma=\gamma^{\prime} / g^{\prime}(f(0))$ such that $\lim _{z \rightarrow 0} \frac{f(z)}{z}=r$. By means of a preliminary linear fractional transformation, the above technique can be modified to apply to any point on the real axis and the theorem is proved.

Let $w=f(z)$ be an interior transformation in the sense of Stoilow in an arbitrary domain $\Omega$, i.e. $w=f(z)$ is continuous and single-valued in $\Omega$, takes open sets in $\Omega$ into open sets in the $w$-plane and does not take any continuum into a single point of the $w$-plane. The set of all pairs of points $(z, f(z))$ forms a Riemann surface $R$, the Riemannian image of $\Omega$ under $f(z)$. It follows from the work of Stoilow [13] that $f(z)$ can be represented in the form $f(z)=$ $\varphi(T(z))$ where $\zeta=T(z)$ is a homeomorphism of $\Omega$ onto a new domain $\Omega^{\prime}$ in the $\zeta$-plane and $w=\varphi(\zeta)$ is an analytic function in $\Omega^{\prime}$. A complex valued function of a complex variable, $w=f(z)$ which is either a constant or an interior transformation in the sense of Stoillow for which the function $\zeta=T(z)$, in the factorization $w=f(z)=\varphi(T(z))$, is a $K$-quasiconformal mapping will be called a $K$-quasiconformal function.

The above definition, that a function $w=f(z)$ is a $K$-quasiconformal function if it admits a representation as $w=f(z)=\varphi(T(z))$ where $\zeta=T(z)$ is a $K$-quasiconformal homeomorphism and $f(\zeta)$ is an analytic function, is not as artificial as it first appears. Indeed, it is possible to give geometric and analytic characterizations of $K$-quasiconformal functions. The following theorems which we 
merely state are examples of such characterizations.

THEOREM: (Geometric characterization of quasiconformal functions)

Let $w=f(z)$ be a sense-preserving interior transformation in the sense of Stoilow in a domain $G$ such that whenever a quadrilateral $Q, \bar{Q} \subset G$ is mapped topologically by $w=f(z)$ onto a quadrilateral $Q^{\prime}$ then $\bmod \left(Q^{\prime}\right) \leqq K \bmod (Q)$, then $w=f(z)$ is a K-quasiconformal function in $G$.

Theorem: (Analytic characterization of $K$-quasiconformal functions)

Let $w=f(z)$ have $L^{2}$-derivatives in a domain $G$, and let $\max _{\theta}\left|D_{\theta} f(z)\right|^{2} \leqq K J(z)$ almost everywhere, then $w=f(z)$ is a K-quasiconformal function in $G$.

We now prove an analogue of Fatou's theorem for $K$-quasiconformal functions.

THEOREM:

If $w=f(z)$ is a bounded K-quasiconformal function in the upper half-plane, $\operatorname{Im}(z)>0,|f(z)| \leqq M$, and if there exists an essentially bounded measurable function $\kappa(z)$ such that for almost all $z=x+i y$,

$$
Q[f(x)]-1 \leqq \kappa(x) \cdot y,
$$

then the limit, $\lim _{y \rightarrow 0} f(x+i y)=f^{*}(x)$, exists for all real numbers $x$ except possibly for an exceptional set of linear measure zero.

Proof: Since the Riemannian image $R$ of $\operatorname{Im}(z)>0$ under $w=f(z)$ is of hyperbolic type, let $\zeta=\varphi^{-1}(w)$ be the function which maps $R$ one-to-one conformally onto $\operatorname{Im}(\zeta)>0$. The inverse function $w=\varphi(\zeta)$ is a bounded analytic function in $\operatorname{Im}(\zeta)>0$. Setting $\zeta=\varphi^{-1}(w)=\varphi^{-1}(f(z))=T(z)$, we obtain the factorization of $w=f(z)=\varphi(T(z))$. The mapping $\zeta=T(z)$ is a $K$-quasiconformal mapping of $\operatorname{Im}(z)>0$ onto $\operatorname{Im}(\zeta)>0$ such that

$$
Q[T(z)]-1 \leqq \kappa(x) \cdot y .
$$

By Fatou's theorem [8], we know that the limit, $\lim _{\eta \rightarrow 0} \varphi(\xi+i \eta)$ exists for all $\xi$ except for a possible set $E$ of points on the $\xi$ axis of measure zero. Because of an extension ${ }^{1 !}$ of Lindelöf's theorem, it follows that $\lim _{y \rightarrow 0} f(x+i y)$ exists for all points $x \notin F=T^{-1}(E)$. In order to prove that $F$ has linear measure zero

1) The proof given in [14] for pseudo-analytic functions can be easily modified to give the same result for the slightly more general class of $K$-quasiconformal functions. 
we have only to note (a well-known fact) that on the real axis the homeomorphism $\zeta=T(z)$ which is a real-valued monotone increasing function with a nonzero finite derivative at each point is absolutely continuous on any closed interval $[a, b]$ and hence the image of a set of measure zero is of measure zero."

In addition to studying the question of existence of radial limits for a quasiconformal function it is also of interest to investigate "how large" the set of radial limit values is. If $w=f(z)$ is a bounded $K$-quasiconformal function in $|z|<1$ the radial limits must exist on a set of points everywhere dense on $|z|=1$.

In 1938, I. I. Privalov ${ }^{2}$ proved the following theorem concerning the set of radial limit values of a meromorphic function.

\section{THEOREM:}

Let $w=f(z)$ be a non-constant meromorphic function in $|z|<1$ and let $A$ be an arc of $|z|=1$. If $w=f(z)$ possesses radial limits on a set $B$ of $|z|=1$ for which $B \cap A$ is both of the second category and metrically dense on the arc $A$, then the set of radial limit values of $f(z)$ contains a set of positive logarithmic capacity.

Cartwright and Collingwood [2] have employed the theory of cluster sets ${ }^{3}$ to extend this result of Privalov and we shall now show how their techniques can be modified to apply to quasiconformal functions.

\section{THEOREM:}

Let $w=f(z)$ be a quasiconformal function defined in $|z|<1$. If the set of points $e^{i \theta}$ on $|z|=1$ at which the complement of the radial cluster set is nonempty is of the second category on some arc $A$ of $|z|=1$, then, unless $f(z)$ is identically constant, the set of radial limit values of $f(z)$ on the arc $A$ is of positive linear measure.

Proof: If $e^{i \beta}$ is a point of $A$ for which the complement of the cluster set $C\left(f, e^{i \beta}\right)$ is non-empty, since the cluster set is a closed set, the complement contains an open disk $\Delta:|w-\alpha|<\delta$ or $(|w|>\delta)$ and therefore there exists a neighborhood $V_{\rho}\left(e^{i \beta}\right)$ of $e^{i \beta}$ of radius $\rho$ such that if $\left|z-e^{i \beta}\right|<\rho$, then $|f(z)-\alpha|$

1) Cf., for example [12], Saks: Theory of the Integral, p. 127, §9, Chapter IV.

2) See Privalov [10] and [11].

3) See Noshiro [9] for a systematic treatment of the boundary behaviour of analytic functions. 
$>\delta(|f(z)|<\delta)$. Thus there is an $\operatorname{arc} B$ on $|z|=1, B=\left\{e^{i \theta}|| e^{i \theta}-e^{i \beta} \mid<\frac{\rho}{2}\right\}$ and a radius $\rho^{\prime}$ such that if $e^{i \theta} \in B$ and $r>\rho^{\prime}$ then $\left|\frac{1}{f\left(r e^{i \theta}\right)-\alpha}\right|<\frac{1}{\delta}\left(\left|f\left(r e^{i \theta}\right)\right|<\delta\right)$.

Let us assume that $f(z)$ itself is bounded in some neighborhood of the arc $B$, i.e., $|f(z)|>\delta$ rather than considering the auxiliary function $\frac{1}{f(z)-\alpha}$ since the two cases are the handled in the same fashion. On the $\operatorname{arc} B$, there exist two points $e^{i \theta_{1}}$ and $e^{i \theta_{2}}$ such that the radial limits

$$
\lim _{r \rightarrow 1} f\left(r e^{i \theta_{1}}\right)=f^{*}\left(e^{i \theta_{2}}\right) \text { and } \lim _{r \rightarrow 1} f\left(r e^{i \theta^{\prime}}\right)=f^{*}\left(e^{\theta i_{2}}\right) \text { exist and } f^{*}\left(e^{i \theta_{1}}\right) \neq f^{*}\left(e^{i 0_{2}}\right) .^{1)}
$$

This is the case because in the factorization $f(z)=g(T(z))$ where $T(z)=\eta$ is a quasiconformal mapping and $w=g(\eta)$ is a bounded analytic function, the radial limits of $g(\eta)$ exists almost everywhere on the $\operatorname{arc} B_{\eta}\left(B_{\eta}\right.$ is the image of the arc $B$ under $\eta=T(z))$ and cannot be constant on a set of positive measure. Thus there exist two points $\eta_{1}, \eta_{2}$ on $|\eta|=1$ such that $g^{*}\left(\eta_{1}\right) \neq g^{*}\left(\eta_{2}\right)$. From the extension of Lindelöf's theorem cited above, it follows that $f^{*}\left(e^{i \theta_{1}}\right)$ $\neq f^{*}\left(e^{i \theta_{2}}\right)$ where $T\left(e^{i \theta_{1}}\right)=\eta_{1}$, and $T\left(e^{i \theta_{2}}\right)=\eta_{2}$.

Since $w=g(\eta)$ is a bounded analytic function in $|\eta|<1$, the projection on the open straight line $L$ between $w_{1}=g^{*}\left(\eta_{1}\right)$ and $w_{2}=g^{*}\left(\eta_{2}\right)$ of the set of radial limit values $g^{*}(\eta)$ with $\eta_{1}$ between $\eta_{1}$ and $\eta_{2}$ on $|\eta|=1$, includes all points of $L^{2)}$, the set of all radial limit values of $f\left(r e^{i \theta}\right)$ for $\theta_{1}<\theta<\theta_{2}$ is of positive linear measure.

The theorem is proved then if it can be established that there exists at least one point $e^{i \beta}$ on the arc $A$ for which the complement of the cluster set $C\left(f, e^{i \beta}\right)$ is non-empty. Collingwood ${ }^{1 \prime}$ proved that for an arbitrary continuous function (real or complex valued) defined in $|z|<1$ the radial cluster set $C_{\rho}\left(f, e^{i \theta}\right)$ coincides with cluster set $C\left(f, e^{i \theta}\right)$ in a residual set of points $e^{i \theta}$ (i.e., the complement of a set of the first category) on $|z|=1$. Hence if on the $\operatorname{arc} A$ the set of points at which the complement of the radial cluster set is non-empty is of the second category, the set of points where the complement of the cluster set is non-empty is also of the second category and therefore is not empty, and the proof is complete.

\footnotetext{
1) We shall denote the radial ${ }_{2}^{*}$ limit ${ }_{s}^{*}$ value of a function $f\left(r e^{i \theta}\right)$ by $f^{*}\left(e^{i \theta}\right)$.

2) See Lemma 4 page 103 of [4].

3) See Theorem 1 in [3].
} 


\section{BIBLICGRAPHY}

[1] Beurling, A. and Ahlfors, L.: The boundary correspondence under quasiconformal mappings. Acta Math. 96 (1956), 125-142.

[2] Cartwright, M. L. and Collingwood, F. F.: The radial limits of functions meromorphic in a circular disc. Math. Zeitschr. 76 (1961), 404-410.

[ 3 ] Collingwood, E. F.: Cluster sets and prime ends. Ann. Acad. Sci. Fenn. Ser. A.1. 250/6 (1958), 1.-11.

[ 4 ] Collingwood, E. F. and Cartwright, M. L.: Boundary theorems for a function meromorphic in the unit circle. Acta Math. 87 (1952), 83-146.

[ 5 ] Künzi, H.: Quasikonforme Abbildungen. Springer-Verlag, Berlin (1960).

[6] Lehto, O.: On the differentiability of quasiconformal mappings with prescribed com. plex dilatation. Ann Acad. Sci. Fenn. Ser. A. I. 275 (1960), 1-27.

[7] Mori, A.: On quasi-conformality and pseudo-analyticity. Trans. Amer. Math. Soc. 84 (1956), 56-77.

[8] Nevanlinna, R.: Eindeutige analytische Funktionen, 2nd Auf. Springer-Verlag, Berlin (1953).

[ 9 ] Noshiro, K.: Cluster sets. Springer-Verlag, Berlin (1960).

[10] Privalov, I. I.: Sur quelques applications de la mesure harmonique des ensembles de points à certains problèmes de la théorie des fonctions. Rec. Math. Moscou, N. s. (3) (Russian; French summary 533) (1938), 527-532.

[11] Privalov, I. I.: Randeigenschaften analytischer Funktionen. (Übersetzung aus dem Russischen), Deutscher Verlag der Wissenschaften, Berlin (1956).

[12] Saks, S.: Theory of the integral, Stechert, New Yörk (1937).

[13] Stoïlow, S.: Leçons sur les principes topologiques de la théorie des fonctions analytiques. Gauthier-Villars, Paris (1938).

[14] Storvick, D. A.: On pseudo-analytic fnnctions. Nagoya Math. J. 12 (1957), 131-138.

\section{Mathematics Research Center}

U.S. Army

University of Wisconsin 